\title{
The properties of strong couple bound polaron in monolayer
}

\author{
graphene \\ Zhao-Hua Ding, Ying Zhao, and Jing-Lin Xiao \\ College of physics and electronic information, Inner Mongolia University for the Nationalities, \\ Tongliao, 028043, People Republic of China
}

\begin{abstract}
Based on the Hamiltonian of the interaction energy between electron on the surface of the graphene and longitudinal acoustic phonon on the surface of the substrate, the paper studies the properties of strong couple polaron in monolayer graphene considering the coulomb doping problem. The conventional Lee-Low-Pine unitary transformation method and linear combination operator method are used to calculate the ground state energy of the polaron. The results show that the ground state energy of the system has a linear relationship with the magnetic field strength, the cut-off wave number, the coulomb bound parameter, the distance between the graphene and the substrates, meanwhile, the ground state energy will split into two branches near the Dirac point.
\end{abstract}

Keywords Bound polaron; monolayer grapheme; Substrates; Dirac point

PACS numbers: 71.38.-k, 73.21.la, 63.20.kd

\section{Introduction}

Graphene is a kind of only one atom thick material, not only it is the thinnest material that people get ,but also it is the most solid material. Because of its unique structure, inspire people's enthusiasm for its research. In recently years, many researchers domestic and overseas have researched properties of graphene from the aspects of theory and experiment. So far the influence of the magnetic field effect and the Rashba effect on properties of the graphene has been discussed, the results show that solutions to the impurity problem which are qualitatively different from those of zero magnetic field has been found by numerical diagonalization of the large Hamiltonian matrices; the properties of the polaron on the electronic structure of zigzag graphene nanoribbon with different width have been researched by a unitary transformation to get an effective Hamiltonian for nanoribbon in the case of considering electron-phonon interaction [1,2]. Apart from these impurity factors, the Coulomb impurity problem in graphene has also been addressed. The result that below the supercritical impurity magnitude within the Wentzel-Kramers-Brillouin (WKB) approximation can solve the problem has been shown. Without impurity the semiclassical energies correctly reproduce the Landau level spectrum. When a given Landau level is given, the relation of the WKB energy and the absolute value of angular momentum in a way which is consistent with the exact diagonalization result [3]. The problem of screening of an electrically charged impurity and the rate of valley relaxation induced by charged impurities in graphene have been also considered and calculated. When Coulomb interaction is neglected, a special model of graphene is applied, the screening charge has a sign opposite to that of the impurity;the valley relaxation rate by solving the Boltzmann equation can be obtained [4,5]. The effects of spin-orbit 
couplings on the electron (hole) $-{ }^{E}{ }_{2 g}$ phonon (The degeneration mode of iTO and LO phonon is called $E_{2 g}$ near G peak in the graphene Raman spectroscopy) interaction in graphene has been investigated. The effects of spin-orbit couplings on electron and hole polaron formation as well as direct-current conductivity, and spin polarizations of charge carriers have been examined. The Frohlich type Hamiltonian has be used to describe the electron-phonon system within the continuum limit [6]. All the possible stable macromolecules that unit charges can form on graphene in magnetic field have been classified. The result that the binding survives the high temperatures has been argued. Which opens the perspective to nanoscopic manipulation of ions on graphene by using macroscopic tools [7]. The key properties of cloaked states in circularly symmetric potentials have been discussed, and cloaking should be observable in quantum corral geometries via scanning tunneling probe measurements [8]. The results of full numerical Hartree-Fock study of coherent and crystalline ground states of the interacting balanced electron-hole graphene systems in small and intermediate separations with each layer occupying up to four lowest lying Landau levels have been reported. It is shown that the capacitance of some crystallized states as well as uniform coherent states are significantly enhanced compared to geometrical values solely due to Coulomb interactions and quantum corrections [9].The problem of an unscreened Coulomb charge in graphene has been addressed and the local density of states and displaced charge as a function of energy and distance from the impurity has been calculated [10].

While most of the results of the study have concerned on electron transport properties on graphene, in fact the other properties of graphene still need to be researched. The magnetopolaron is studied in the presence of a magnetic field in monolayer graphene. The phenomenon the energy gap will be opened in the zero-energy Landau level due to the effect of polaron has been found. And the result the EG depends on square-root forms of the magnetic field strength is consistent with recent experimental measurements[11]. But the study on the phonon effect of polaron properties in monolayer graphene is rare. Because of this reason when different substrates are considered, the study on the phonon effect of polaron properties in monolayer graphene is less.Recently the polaron effects are investigated in the presence of a magnetic field based on the carrier-surface optical phonon coupling induced by the polar substrates under the graphene [12]. Meanwhile the reseach also become a hot reseach topic. But so far the research of the phonon effect on the coulomb impurity problem has almost no in monolayer graphene with different substrate. In this paper, in the case of considering the coulomb impurity problem we study the phonon effect of polaron in monolayer graphene with different substrate by using the linear combination operator and the Lee-Low-Pine variational methods. The results that we obtained are in agreement with the cyclotron resonance experiment results.

\section{Theoretical Model and Calculations}

This model assume single layer graphene is sandwiched between the substrate and the air, the strong interaction between longitudinal acoustic phonon on the surface of the substrate and the electronic (hole) in the graphene happens, and this system is supplied a perpendicular and uniform magnetic field. Its Hamiltonian is as follows: 


$$
\begin{aligned}
& H=H_{e}+H_{p h}+H_{e-p h}-\frac{e^{2}}{4 \pi \varepsilon \boldsymbol{r}} \\
& H_{e}=V_{F}\left(\begin{array}{cc}
0 & \pi_{x}-i \pi_{y} \\
\pi_{x}+i \pi_{y} & 0
\end{array}\right) \\
& \pi_{x}=\left(p_{x}-e B y / 2\right) \\
& \pi_{y}=\left(p_{y}+e B x / 2\right) \\
& H_{p h}=\sum_{k, v} \hbar \omega_{s o, v} a_{k}^{+} a_{k} \\
& H_{e-p h}=\sum_{k, v} M_{k, v}\left(a_{-k}^{+}+a_{k}\right) e^{i k \llbracket r} k \\
& M_{k, v}=\sqrt{\left(Q^{2}-\eta \hbar \omega_{s o, v}\right) /\left(2 \varepsilon_{0} k\right)} e^{-k z}
\end{aligned}
$$

Here $\eta=\left(k_{0}-k_{\infty}\right) /\left[\left(k_{\infty}+1\right)\left(k_{0}+1\right)\right], k_{\infty}$ represents the the high frequency dielectric constant, $k_{0}$ represents the the low frequency dielectric constant, $\varepsilon_{0}$ represents the vacuum dielectric constant, $\eta$ represents the dielectric constant of the substrate, $\omega_{s a, v}$ represents the surface acoustic phonon frequencies, the of the creation and annihilation linear combination operators $b_{j}^{+}$and $b_{j}[13]$ are represented as:

$$
\begin{gathered}
p_{j}=(\hbar \lambda / \sqrt{2})\left(b_{j}^{+}+b_{j}\right) \\
r_{j}=(i / \sqrt{2} \lambda)\left(b_{j}-b_{j}^{+}\right)
\end{gathered}
$$

In the formula (8) and (9), $\lambda=\sqrt{e B / 2 \hbar}$, the coulomb bound potential's Fourier expansion in formula (1) can be written as:

$$
\begin{aligned}
& \frac{1}{\boldsymbol{r}}=\frac{2 \pi}{A} \sum_{k} \frac{1}{k} \exp (-i k \cdot \boldsymbol{r}) \\
& U=\exp \left(\sum_{k} f_{k} a_{k}^{+}-f_{k}^{*} a_{k}\right)
\end{aligned}
$$

$A$ presents the graphene area in the formula (10). Then after the Lee-Low-Pine unitary transformation [14], the Hamiltonian form $H^{\prime}=U^{-1} H U$ can be got, the wave functions of the system has been shown as:

$$
\left|\psi_{n}\right\rangle|0\rangle=\frac{1}{\sqrt{2}}\left(\begin{array}{c}
C_{n}^{\prime}|n-1\rangle|0\rangle \\
C_{n}|n\rangle|0\rangle
\end{array}\right)
$$


Where $C_{n}^{\prime}=1-\delta_{n, 0}, \quad C_{n}=\sqrt{1+\delta_{n, 0}}, b_{j}^{+}|n\rangle=\sqrt{n+1}|n+1\rangle, \quad b_{j}|n\rangle=\sqrt{n}|n-1\rangle .|0\rangle$ is the zero-phonon state without perturbation, $a_{k}|0\rangle=0$, when $n=0$,so the expectation of the ground energy $E_{0}=\left\langle 0\left|\left\langle\psi_{0}\left|H^{\prime}\right| \psi_{0}\right\rangle\right| 0\right\rangle$ of the system is obtained and the variational method is supplied to the expectation about $f_{k}^{*}$ and $f_{k}$, so the ground state energy of the system can be expressed as:

$$
\begin{gathered}
E_{0 \pm}=\mp\left[\sum_{k, v}\left[\frac{\left|M_{k, v} \exp \left(-\frac{k^{2}}{4 \lambda^{2}}\right)\left(1+\frac{k^{2}}{4 \lambda^{2}}\right)\right|^{2}}{\hbar \omega_{s o, v}}+\frac{2 \pi g}{A} \frac{1}{k} \exp \left(-\frac{k^{2}}{4 \lambda^{2}}\right)\left(1+\frac{k^{2}}{4 \lambda^{2}}\right)\right]\right. \\
=\mp\left[\sum_{v} \int_{0}^{k_{c}} \frac{Q^{2} \eta \hbar \omega_{s o, v} e^{-2 k z_{0}} e^{-\frac{k^{2}}{2 \lambda^{2}}}\left(1+\frac{k^{2}}{4 \lambda^{2}}\right)^{2}}{4 \pi \varepsilon_{0} \hbar \omega_{s o, v}} d k+\int_{0}^{k_{c}} 2 \pi g \cdot e^{\left(-\frac{k^{2}}{4 \lambda^{2}}\right)}\left(1+\frac{k^{2}}{4 \lambda^{2}}\right) d k\right]
\end{gathered}
$$

Where $g=\frac{e^{2}}{16 \pi^{3} \varepsilon}, \varepsilon$ presents the dielectric constant and the $z_{0}$ presents the distance of between graphene and the substrate.

\section{Numerical results and discussion}

In the process of calculation, to make the numerical calculation convenient, these parameters in the table below are adopted, and the results are showed in Figs 1-4.

Table of the Experimental parameters used in the numerical calculations

\begin{tabular}{cccc}
\hline Quantity(units) & $\mathrm{SiC}$ & $\mathrm{HfO}_{2}$ & $h-B N$ \\
\hline$k_{0}\left(\varepsilon_{0}\right)$ & 9.7 & 22.0 & 5.1 \\
$k_{\infty}\left(\varepsilon_{0}\right)$ & 6.5 & 5.0 & 4.1 \\
$\hbar \omega_{s o, 1}(\mathrm{meV})$ & 116 & 19 & 101 \\
$\hbar \omega_{s o, 2}(\mathrm{meV})$ & 167 & 53 & 195 \\
\hline
\end{tabular}




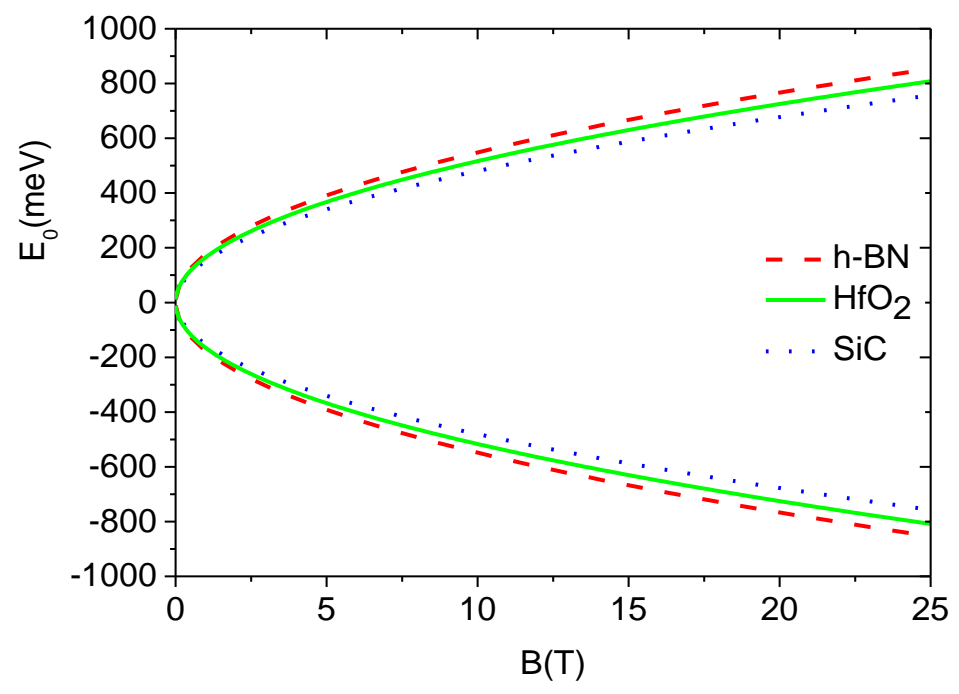

Fig. 1 The relation curves between the ground state energy and the magnetic field strength.

Fig.1 describes the variational curve of the system's ground-state energy and the magnetic field strength, when the distance between the substrates and graphene $z_{0}=1 \mathrm{~nm}$, coulomb potential parameter $\mathrm{g}=0.2$, cut-off wave number $k_{c}=7.6 \times 10^{9} \mathrm{~m}^{-1}$. For three different substrates, the ground-state energy of the graphene will be split into two symmetrical band which will increase with increasing the magnetic field strength gradually. That is because electron and hole are coexisting on landau energy level in the graphene. Besides, this system is influenced by the Lorentz force, electrons and holes will be separated into the opposite direction, and they will interact at the same time with phonon and electron on the surface of substrate, the interaction energy of electron- phonons is negative, the interaction energy of the hole-phonon is positive, but the absolute value of the energy is equal, interaction energy of electron- electron is also negative.

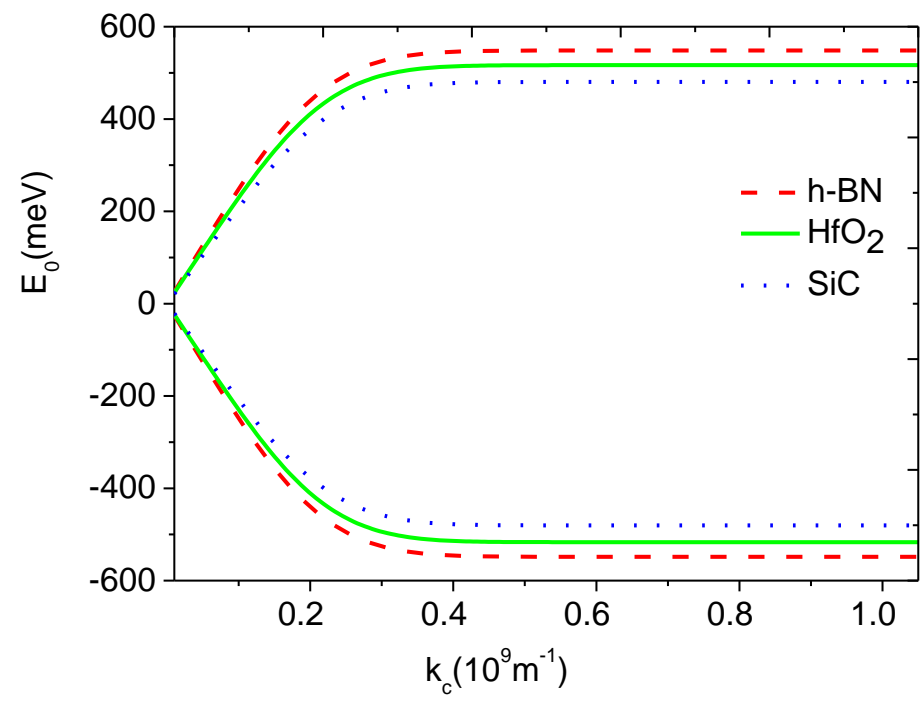


Fig. 2 The relation curves between the ground state energy and the cut-off wavenumbers.

Fig. 2 shows when $B=10 \mathrm{~T}, \mathrm{~g}=0.2, z_{0}=1 \mathrm{~nm}$, the ground-state energy changes with the cut-off wave number. We can see the ground state energy increases with the increasing of the cut-off wavenumber and, finally the energy will reach a stable value. Results are approximate with the results of the cyclotron resonance experiment.

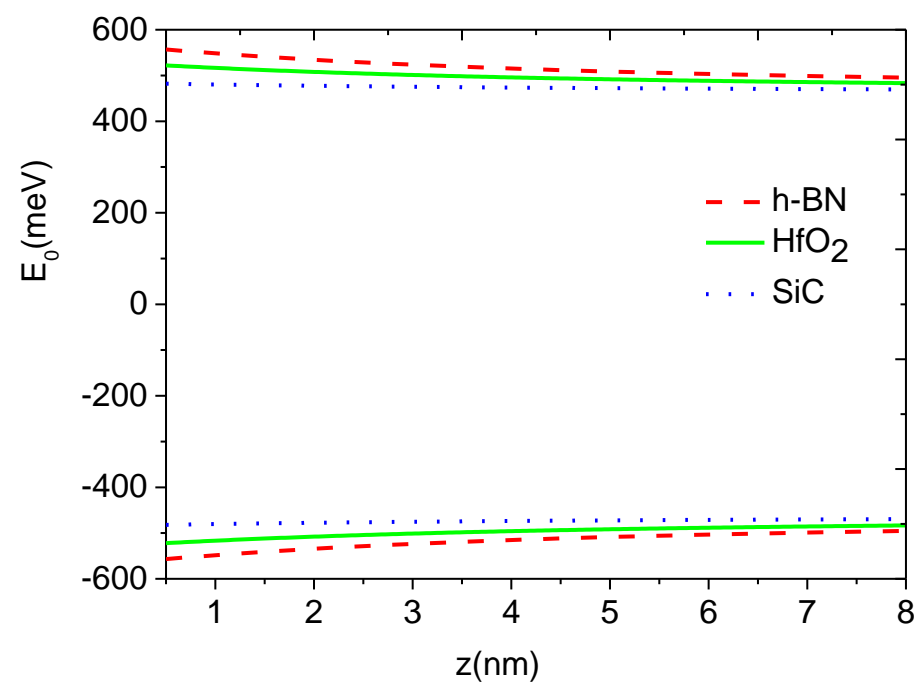

Fig. 3 The relation curves between the ground state energy and the basal spacing.

Fig. 3 indicates when $\mathrm{B}=10 \mathrm{~T}, \mathrm{~g}=0.2, k_{c}=7.6 \times 10^{9} \mathrm{~m}^{-1}$, the strong coupling bound polaron ground state energy decreases with the increasing of substrate spacing. The reason of this result is that as the increase of substrate spacing, the Coulomb interaction becomes weak, so that the ground state energy decreases.

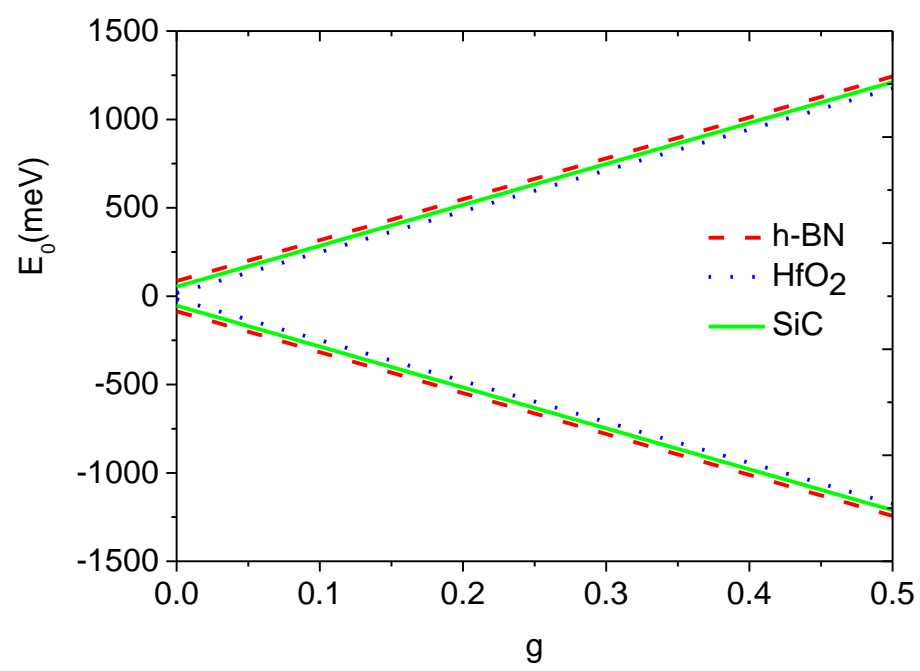

Fig. 4 The relation curves between the ground state energy and the Couloumb bound potential. 
Fig.4 presents the result that the ground-state energy of strong coupling bound polaron will increase with increasing the Coulomb bound potential parameter in the monolayer grapheme. The reason is that the Coulomb bound potential will increase with increasing the Coulomb bound parameters, and the size of the ground state energy depends on the Coulomb bound potential, so the ground state energy increases with the Coulomb bound potential.

\section{Conclusions}

This paper studies the properties of monolayer graphene's polaron by variational method and unitary transformation method. When the Coulomb interaction of the electron on the surface of the monolayer graphene and electron in the substrate is considered, the ground state energy of strong coupling bound polaron are calculated. Results show that the ground state energy of the system increases with increasing the magnetic field strength, decreases gradually with increasing the substrate spacing, gradually increases with increasing the cut-off wavenumber and finally reaches a stable value, and decreases with increasing the coulomb bound parameters.

\section{Acknowledgement}

This project was supported by the National Science Foundation of China under Grant No. 11464033.

\section{References}

[1]S.C. Kim and S. R. E. Yang, Ann. Phys. 347, 21 (2014)

[2]M. Modarresi , A. Mogulkoc , M.R. Roknabadi , and N. Shahtahmasebi, Physica E 66303 (2015)

[3]Y. Zhang, Y. Barlas, and K. Yang, Phys. Rev. B 85, 165423 (2012)

[4]R. R. Biswas, S. Sachdev, and D. T. Son, Phys. Rev. B 76, 205122 (2007)

[5]P. Boross and A. Palyi, Phys. Rev. B 92, 035420 (2015)

[6]A. Mogulkoc, a, M. Modarresi, and B.S. Kandemir, Eur. Phys. J. B 88 49(2015)

[7]S. Slizovskiy, Phys. Rev. B 92, 195426 (2015)

[8]T. Stauber and N. M. R. Peres, J. Phys.:Condens.Matter 20, 055002 (2008)

[9]B. Roostaei, Phys. Rev. B 92, 245102 (2015)

[10]V. M. Pereira, J. Nilsson, and A. H. C. Neto, Phys. Rev. Lett. 99, 166802 (2007)

[11]W. P. Li, Z. W. Wang, J. W. Yin, and Y. F. Yi, J. Phys.: Condens. Matter 24, 135301 (2012)

[12]Z. W. Wang, L. Liu, and Z. Q. Li, Appl. Phys.Lett.106, 101601 (2015)

[13] W. J. Huybrechts, J. Phys.C: Solid State Phys. 10, 3761 (1977)

[14]T. D. Lee, F. E. Low, and D. Pines, Phys. Rev. 90, 297 (1953) 